\title{
Topographic distribution of the tibial somatosensory evoked potential using coherence
}

\author{
D.B. Melges, A.F.C. Infantosi and A.M.F.L. Miranda de Sá \\ Programa de Engenharia Biomédica, Instituto Alberto Luiz Coimbra de Pós-graduação e Pesquisa de \\ Engenharia, Universidade Federal do Rio de Janeiro, Rio de Janeiro, RJ, Brasil
}

Correspondence to: D.B. Melges, Programa de Engenharia Biomédica, Universidade Federal do Rio de Janeiro, Caixa Postal 68510, 21941-972 Rio de Janeiro, RJ, Brasil

Fax: +55-21-2562-8591. E-mail: dmelges@peb.ufrj.br

\begin{abstract}
The objective of the present study was to determine the adequate cortical regions based on the signal-to-noise ratio (SNR) for somatosensory evoked potential (SEP) recording. This investigation was carried out using magnitude-squared coherence (MSC), a frequency domain objective response detection technique. Electroencephalographic signals were collected (International 10-20 System) from 38 volunteers, without history of neurological pathology, during somatosensory stimulation. Stimuli were applied to the right posterior tibial nerve at the rate of $5 \mathrm{~Hz}$ and intensity slightly above the motor threshold. Response detection was based on rejecting the null hypothesis of response absence (significance level $\alpha=0.05$ and $M=500$ epochs). The best detection rates (maximum percentage of volunteers for whom the response was detected for the frequencies between 4.8 and $72 \mathrm{~Hz}$ ) were obtained for the parietal and central leads mid-sagittal and ipsilateral to the stimulated leg: C4 (87\%), P4 (82\%), $\mathrm{Cz}$ (89\%), and Pz (89\%). The P37-N45 time-components of the SEP can also be observed in these leads. The other leads, including the central and parietal contralateral and the frontal and fronto-polar leads, presented low detection capacity. If only contralateral leads were considered, the centro-parietal region ( $\mathrm{C} 3$ and $\mathrm{P} 3$ ) was among the best regions for response detection, presenting a correspondent well-defined N37; however, this was not observed in some volunteers. The results of the present study showed that the central and parietal regions, especially sagittal and ipsilateral to the stimuli, presented the best SNR in the gamma range. Furthermore, these findings suggest that the MSC can be a useful tool for monitoring purposes.
\end{abstract}

Key words: Somatosensory response detection; Magnitude-squared coherence; Tibial nerve; Topography

Research supported by CAPES (PROEX - \#308/2008), CNPq (\#472350/2007-2), and FAPERJ (\#E-26/100.387/2008, \#E-26/ 171.113 and \#152.797/2006).

Received December 19, 2007. Accepted November 14, 2008

\section{Introduction}

The somatosensory evoked potential (SEP) is the electrical response of the nervous system to a sensory stimulation. The tibial nerve SEP (tSEP), elicited by an electrical stimulation, has been widely used in neurophysiologic evaluation, especially for diagnostics and monitoring surgeries (1). The diagnosis (or prognosis) is based on the latency and amplitude of tSEP characteristic peaks. Furthermore, during surgery monitoring, the surgical strategy can be modified based on amplitude decrease or latency increase of the tSEP components. However, this exam has been reported to be a subjective procedure that depends on both the quality of the electroencephalogram (EEG) recording and the expertise of the observer in identifying the tSEP peaks. Hence, the application of statistical techniques $(2,3)$ has been used to overcome the subjectivity of the morphological tSEP analysis. These techniques, known as objective response detection (ORD), allow inference about the absence of stimuli-response with a constant false-alarm rate, which is the significance level of the statistical test (4-6).

Although both morphological analysis and ORD have been applied successfully to the detection of cortical re- 
sponses to a sensory stimulation, the methodological fundamentals of tSEP recording are still an object of discussion. The loci for the registration of the most well-defined and highest amplitude tSEP remain in debate. Moreover, the interpretation of the tSEP components and their relation to anatomical structures is still controversial (7-10). Furthermore, the nomenclature used by investigators in the area has been inconsistent and can lead to confusing interpretations, particularly for bipolar derivation that involves more than one site of electrical activity.

The bipolar derivation [Cz'-Fpz'] has been recommended by Misulis (11) for the registration of the parietal complex P37-N45. Similarly, Chiappa (1) suggests the use of [Fz-Cz] (or [Fz-mid $\mathrm{Cz} / \mathrm{Pz}]$ ) for the identification of the N/P37 (the P37 preceded by a negative peak), which would be a combination of a negative peak in $\mathrm{Fz}$ with a positive one in $\mathrm{Cz}$. In fact, bipolar derivations that include vertex (or near vertex) referenced to forehead leads are very common. This is mainly due to the International Federation of Clinical Neurophysiology (IFCN) Committee report (12) that recommended the use of $\mathrm{Cz}^{\prime}$ ( $2 \mathrm{~cm}$ behind $\mathrm{Cz}$ ) or $\mathrm{Cpz}$ (half way between $\mathrm{Cz}$ and $\mathrm{Pz}$ ) with the reference in $\mathrm{Fz}$.

Another very commonly used bipolar derivation is the [Cc-Ci] (where $\mathrm{Cc}$ is positioned $2 \mathrm{~cm}$ posterior to $\mathrm{C} 3$ or $\mathrm{C} 4$, depending on what is contralateral to the stimulated leg, and $\mathrm{Ci}$ is positioned ipsilateral in a similar way). This derivation is also recommended by the IFCN report (12) and Chiappa (1) and is capable of recording the potentials P37 and N45 of tSEP.

However, the most prominent and consistent scalp SEP-positive deflection, the P37, could be better visualized in other derivations, which has led some investigators to suggest new recording montages $(9,13)$. The choice of a suitable montage is very important, since it directly affects both the interpretation of visual analysis and detection based on statistical techniques such as ORD. In fact, this choice involves the selection of the scalp regions with the highest signal-to-noise ratio (SNR). This is a critical consideration, especially in surgical monitoring, in which the speed of detection of changes in tSEP can avoid negative neurological consequences.

Thus, we investigated the topographical distribution of the SEP by means of a frequency-domain ORD technique, magnitude-squared coherence (MSC) (2), which takes into account both power and phase relationship between EEG and stimulus.

\section{Material and Methods}

\section{Magnitude-squared coherence}

The MSC represents the portion of the squared mean value of the measured EEG that can be explained by the stimulation. The MSC for the discrete-time, finite duration and windowed signal can be estimated as described in Ref. 5. When the stimulus is periodic (such as the pulse train in the present research), the MSC estimate depends only on the measured EEG and may be expressed as (5):

$$
\hat{\kappa}^{2}(f)=\frac{\left|\sum_{i=1}^{M} Y_{i}(f)\right|^{2}}{M \sum_{i=1}^{M}\left|Y_{i}(f)\right|^{2}} \quad \text { (Equation 1) }
$$

where "^" denotes estimate, $Y_{i}(f)$ is the Fourier transform of $\mathrm{i}^{\text {th }}$ window of EEG signal and $M$ is the number of epochs.

Mathematically, the MSC can be related to the SNR ( $\hat{S}$ : the ratio between the power of signal and that of the noise) at any frequency $f$ by (14):

$$
\hat{\kappa}^{2}(f)=\frac{\hat{S}(f)}{\hat{S}(f)+1} \quad \text { (Equation 2) }
$$

Hence, it is clear that $\hat{\kappa}^{2}$ is bounded between 0 and 1 , approaching this latter value as $\hat{S}$ increases (i.e., $\hat{\kappa}^{2}$ tends to the unity when $\hat{S}$ tends to the infinite). On the other hand, when the SNR is very low, the MSC estimate tends to zero. Figure 1 illustrates the behavior of MSC by varying the SNR. Further details about the interpretation of MSC are provided in Ref. 2.

For the estimate of MSC, if we assume that there is no response to the stimulation or, in other words, that no power of the measured EEG is due to the stimulation, we can establish a hypothesis test with the null hypothesis $(\mathrm{H} 0)$ of response absence $\left(\hat{\kappa}^{2}(f)=0\right)$. For the case of no stimuli-response, we can calculate the critical value $\left(\hat{K}^{2}\right.$ crit $)$ analytically for a given significance level $(\alpha)$ and $M$ EEG epochs by (15):

$$
\hat{\kappa}_{c r i t}^{2}=1-\alpha^{\frac{1}{M-1}} \quad(\text { Equation 3) }
$$

The critical value constitutes a detection threshold. That is, if the estimate value $\hat{\kappa}^{2}(f)$ exceeds $\hat{\kappa}_{\text {crit }}^{2}$, we reject the hypothesis of response absence $\mathrm{HO}$ and assume that there is cortical response to the stimulation.

\section{Acquisition of EEG}

EEG was collected during somatosensory stimulation from 38 adult volunteers, 29 men and 9 women (range: 21 to 41 years; mean \pm SD: $28.7 \pm 4.7$ years), and without a history of neurological pathology. The signals were collected using the EEG BNT-36 (EMSA, Brazil, 
www. emsamed.com.br) according to the International 1020 System and all leads were referenced to the earlobe average. The volunteers were in the supine position with their eyes closed during testing. The stimuli were applied by means of current pulses ( $200 \mu \mathrm{s}$ width) to the right posterior tibial nerve using the Atlantis Four (EMSA). The intensity was adjusted to slightly above the motor threshold level (the lowest intensity that produces finger oscillations) at the rate of $5 \mathrm{~Hz}\left(f_{e}\right)$. The ground electrode was positioned on the popliteal fossa. Surface silver electrodes were used for both recording and stimulation. The Ethics Committee of the Hospital Universitário Clementino Fraga Filho of the Federal University of Rio de Janeiro (CEPHUCFF/UFRJ) approved this research and all volunteers gave written informed consent to participate.

\section{Pre-processing}

The signals were first band-filtered within $0.5-100 \mathrm{~Hz}$ and digitized with BNT-36 (16-bits resolution) at the sampling rate of $600 \mathrm{~Hz}$. The EEG signals were segmented into epochs of $208 \mathrm{~ms}$ (spectral resolution of $4.8 \mathrm{~Hz}$ ) synchronized with the stimulation. The averaged signal (SEP) often contains a wideband and stimuli-synchronized noise that is called stimulus artifact, which produces distortion in the frequency domain. The morphology of this signal is characterized by a transient profile, that is, it has high initial amplitude (it can be much higher than the SEP amplitude) that rapidly decays. Tierra-Criollo and Infantosi (3) reported that the more important effect of this artifact occurs up to $5 \mathrm{~ms}$. Hence, in order to avoid the interference of this artifact in the MSC we have set the first $5 \mathrm{~ms}$ after each stimulus to zero. Also, the final $5 \mathrm{~ms}$ were zero padded to ensure window symmetry. Furthermore, a Tukey window with $7 \mathrm{~ms}$ rising (falling) time has been applied to each epoch to assure that the late components of the artifact are also attenuated. Noisy epochs were next discarded by a semi-automatic artifact rejection algorithm, which rejects epochs with more than $5 \%$ of continuous samples or more than $10 \%$ of samples exceeding \pm 3 SD (where SD is the standard deviation of $20 \mathrm{~s}$ of noise-free background EEG selected as reference signal). Both the windowing and the artifact rejection are described in more detail by Infantosi et al. (2). Then, $\hat{\kappa}^{2}(f)$ and $\hat{\kappa}^{2}$ crit were calculated for the acquired signals using Equations 1 and 3 with $\alpha=5 \%$ and $M=500$.

\section{Results}

The SEP is illustrated in Figure 2 for volunteer \#5 (right-handed, stimulated at $22 \mathrm{~mA}$ ). The positions of the SEP traces are in accordance with the electrode localiza- tions in the International 10-20 System. In this figure, we note that the short latency tSEP components were identified mainly in the frontal, central and parietal regions. The potentials were more evident in the midline and ipsilateral to the stimulated leg. This pattern of potential distribution was observed for 23 from the 38 volunteers.

Figure 3 shows another profile of potential distribution that is wider. In this figure, for volunteer \#1 (left-handed, stimulated at $23 \mathrm{~mA}$ ), we observed SEP not only in frontal, central and parietal leads, but also in temporal, occipital and fronto-polar leads. The potentials were more pronounced in the same regions that were noted for the other profile (frontal, central and parietal) for this pattern, which was seen for 15 volunteers.

The result of the application of MSC to the EEG of volunteer \#5 is illustrated in Figure 4. As can be seen, the detection $\left(\hat{\kappa}^{2}(f)>\hat{\kappa}^{2}\right.$ crit, $)$ is observed mainly in the leads $\mathrm{Cz}, \mathrm{Pz}, \mathrm{C} 4$, and $\mathrm{P} 4$ and also with lower MSC values in Fz, $\mathrm{F} 4, \mathrm{~F} 8, \mathrm{~T} 4$, and T6. That is, the MSC values are higher in the same regions where the short latency tSEP components were easily visualized (frontal, central and parietal regions in the mid-sagittal and ipsilateral leads), as can be seen in Figure 2. This observation can be extended to all individuals with this profile.

For volunteer \#1, the MSC result is presented in Figure 5 . In this figure, we can observe that the widespread distribution of SEP, observed in Figure 3, is also reproduced in the topographical distribution of potential detection. Although it is more consistent in the frontal, central and parietal regions, it was possible to detect stimuli-response in temporal (T3, T4, T6), fronto-polar (Fp1) and occipital (Oz and O2) leads. The other volunteers with this pattern of potential

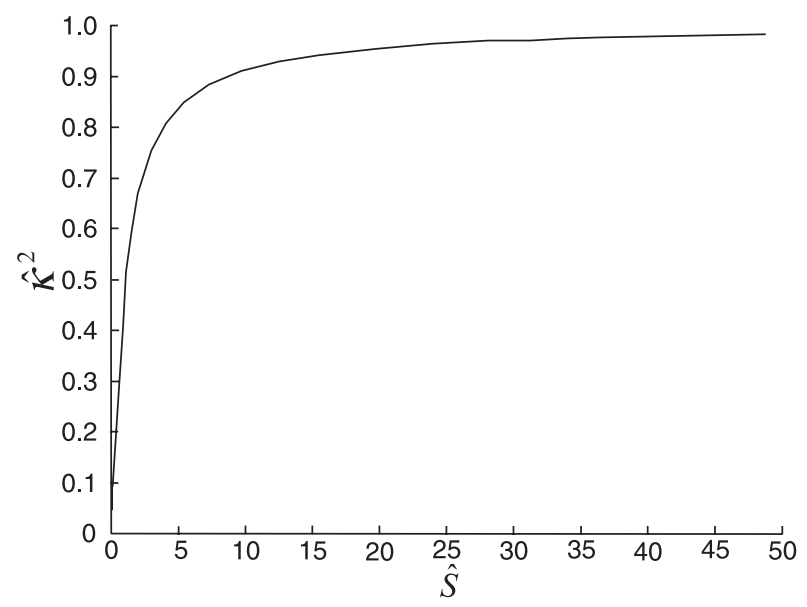

Figure 1. Simulation of the magnitude-squared coherence estimates $\left(\hat{K}^{2}\right)$ for different signal-to-noise ratios $(\hat{S})$. Both variables are dimensionless. 


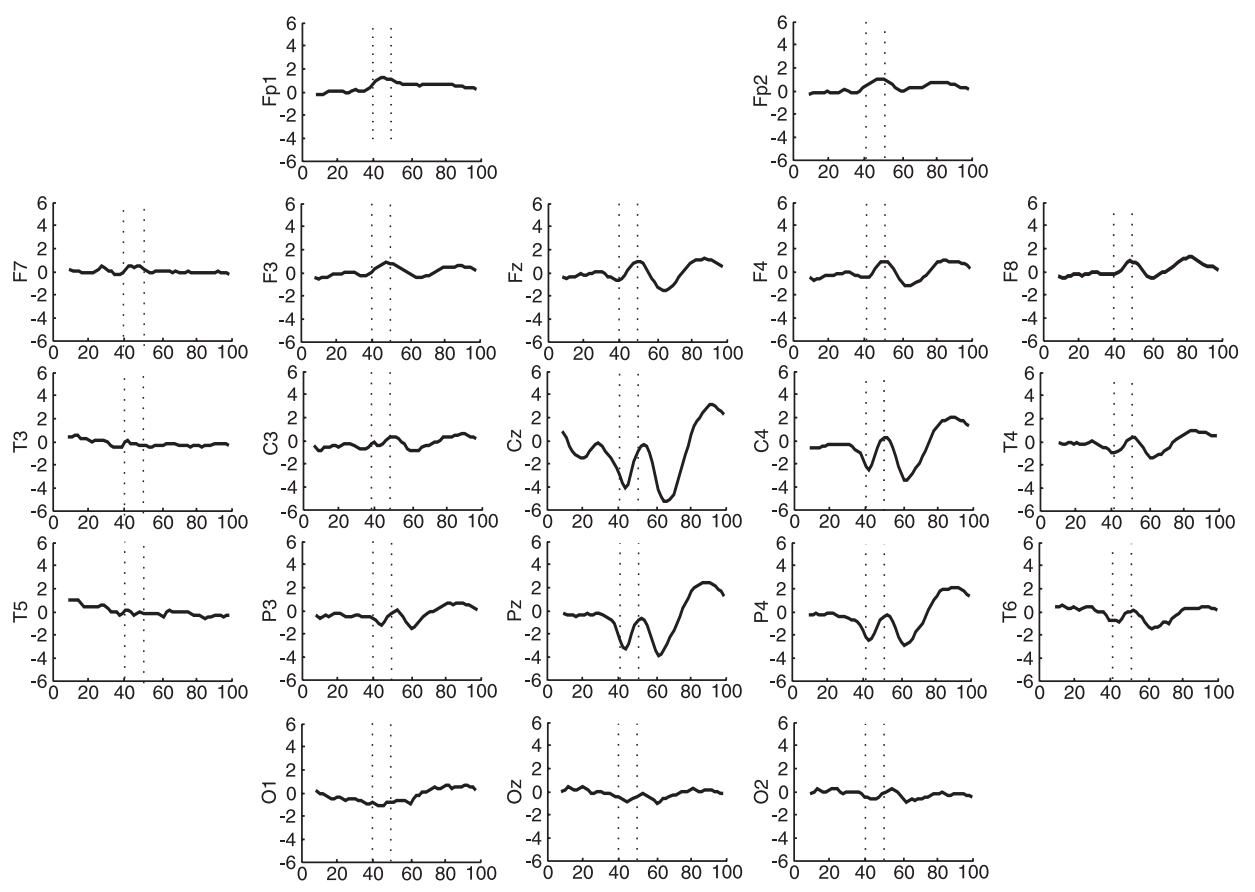

Figure 2. Somatosensory evoked potential topography of volunteer \#5 stimulated at $22 \mathrm{~mA}$. Time scale (abscissa) in ms and amplitude scale (ordinate) in $\mu \mathrm{V}$. Vertical dotted lines indicate 40 and $50 \mathrm{~ms}$, respectively.

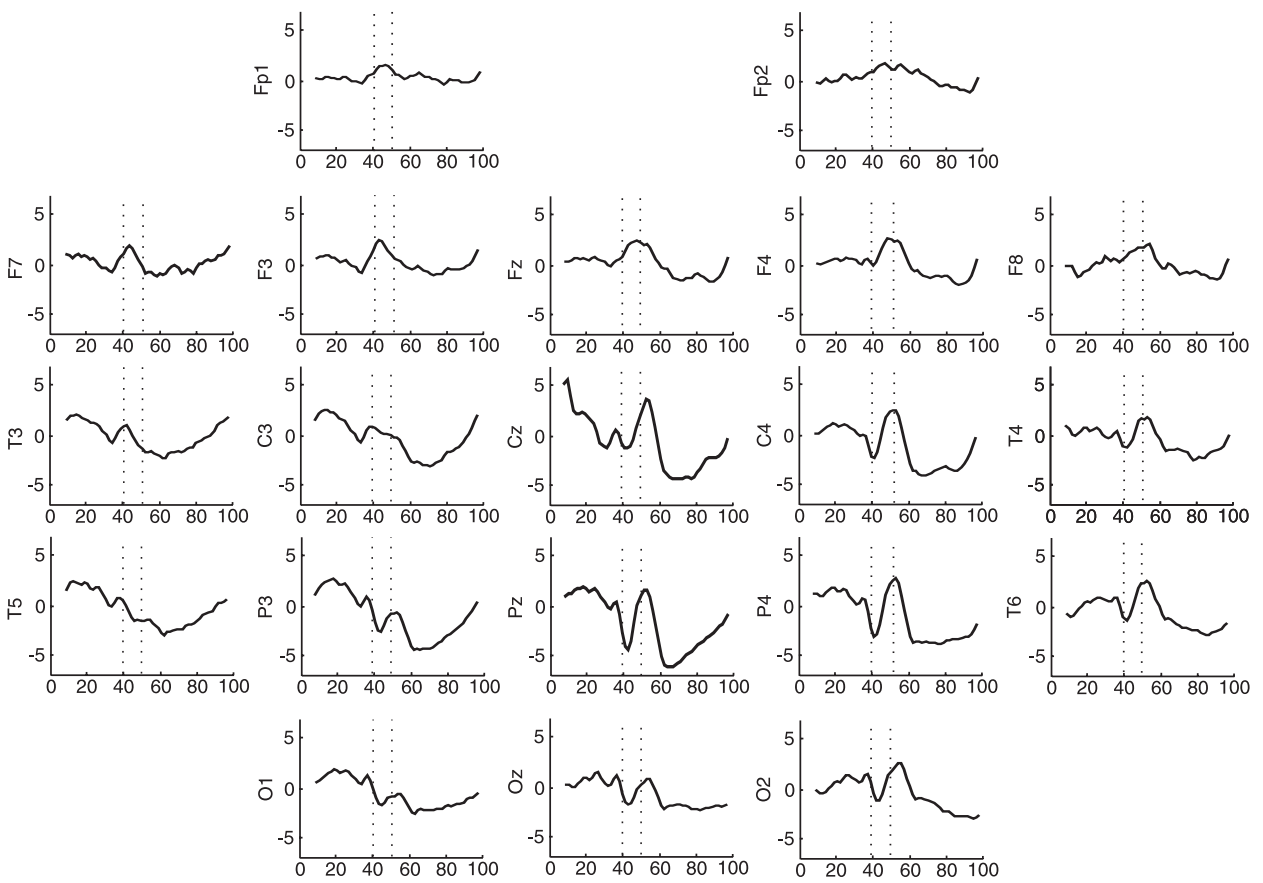

Figure 3. Somatosensory evoked potential topography of volunteer \#1 stimulated at $23 \mathrm{~mA}$. Time scale (abscissa) in ms and amplitude scale (ordinate) in $\mu \mathrm{V}$. Vertical dotted lines indicate 40 and $50 \mathrm{~ms}$, respectively. 


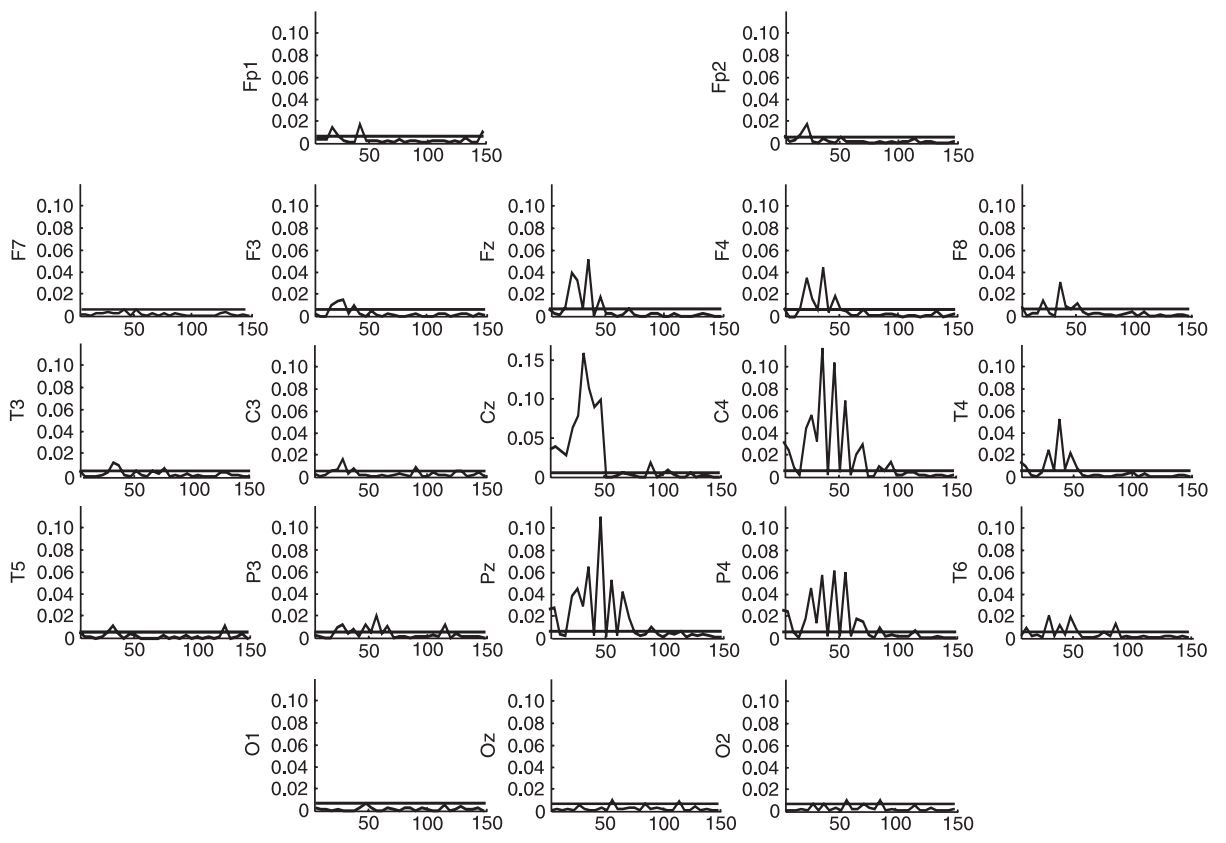

Figure 4. Magnitude-squared coherence (MSC) topography for volunteer \#5. The abscissa is the frequency (Hz) and the ordinate is the MSC (dimensionless). Horizontal line is the critical value $\hat{\kappa}_{\text {crit }}^{2}=0.006$, for $\alpha=5 \%$ and $M=500$.

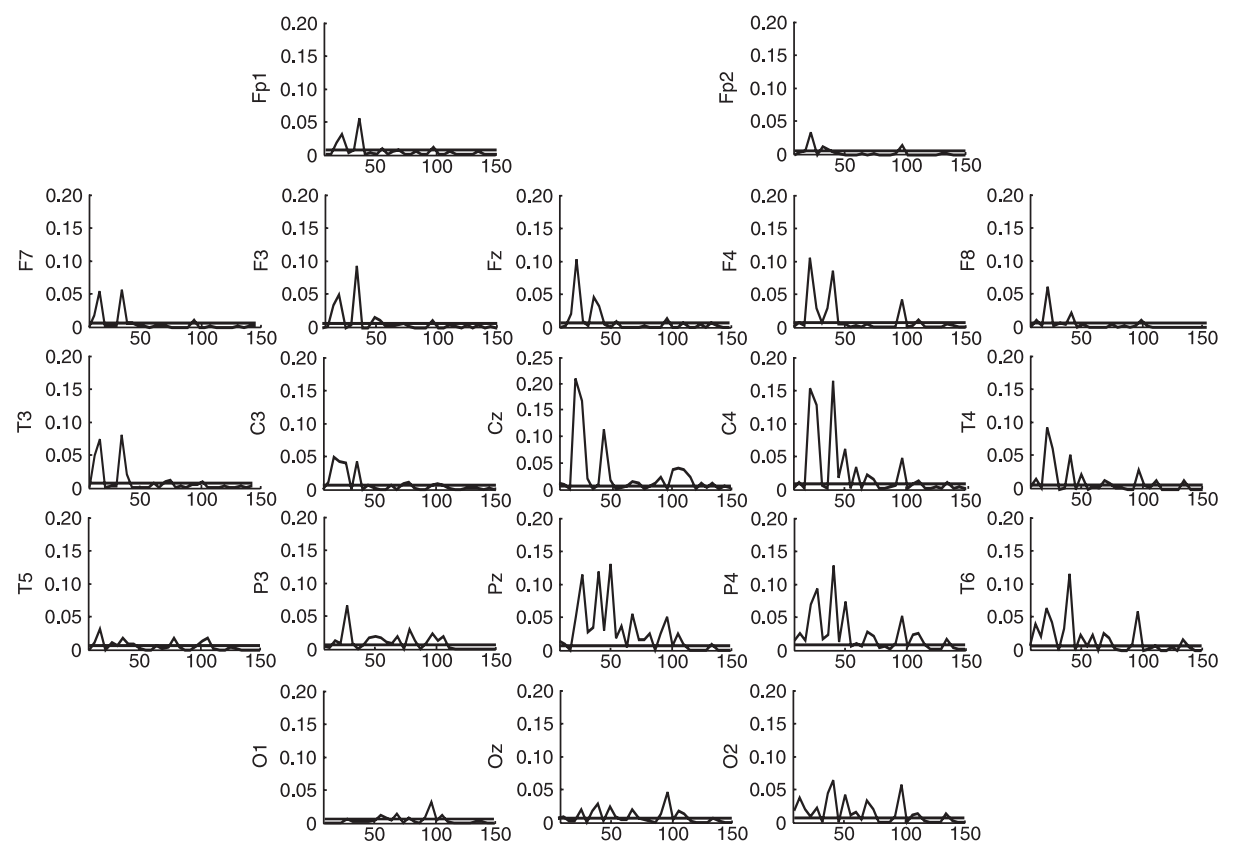

Figure 5. Magnitude-squared coherence (MSC) topography for volunteer \#1. The abscissa is the frequency (Hz) and the ordinate is the MSC (dimensionless). Horizontal line is the critical value $\hat{\kappa}_{\text {crit }}^{2}=0.006$, for $\alpha=5 \%$ and $M=500$. 

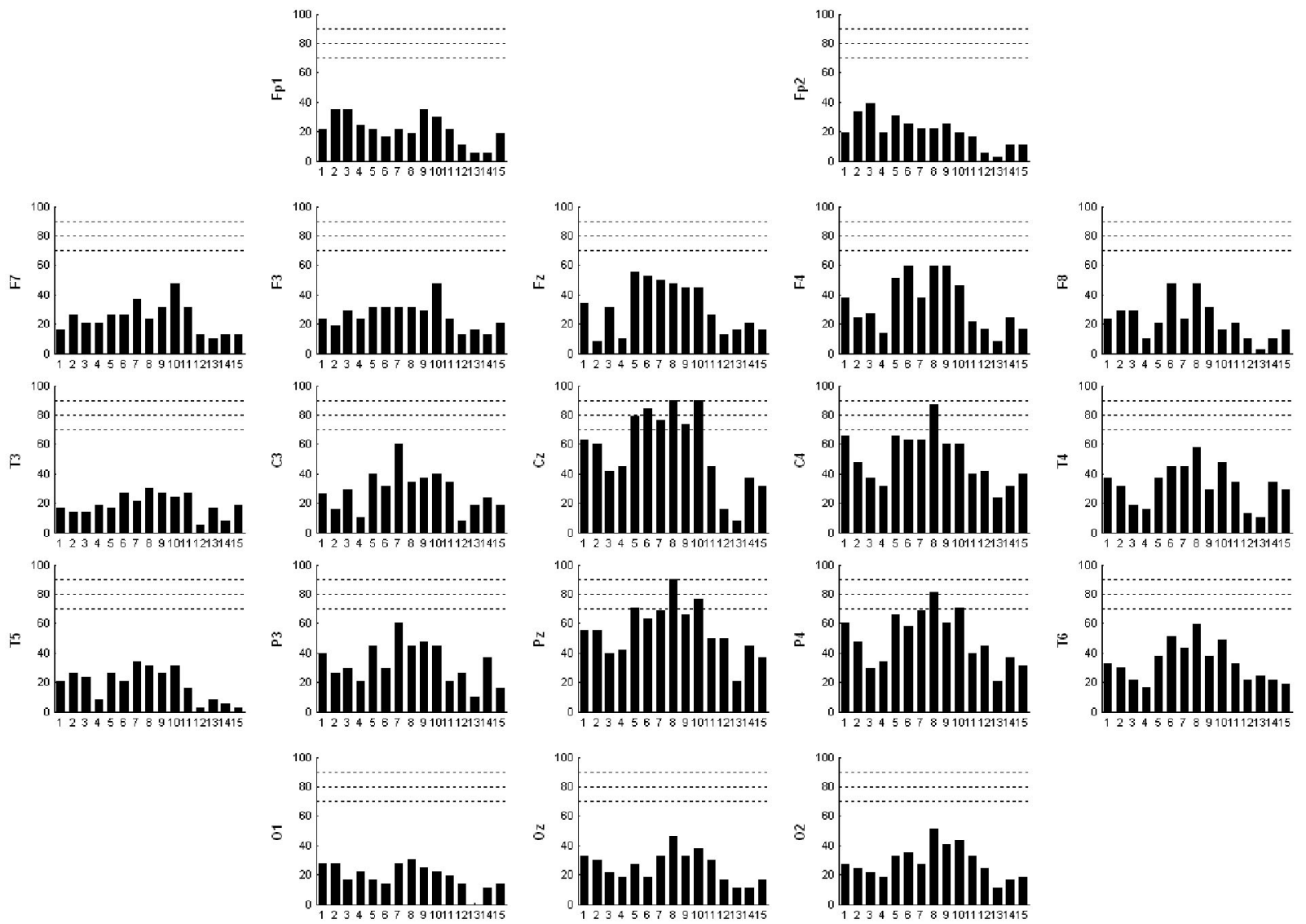

Figure 6. Bar graphics displaying the percentage of volunteers whose response to stimulation could be detected for the $1^{\text {st }}$ to $15^{\text {th }}$ multiples of the stimulation frequency $(5$ to $75 \mathrm{~Hz})$. Horizontal lines indicate 70,80 and $90 \%$ of detection. For derivations $\mathrm{Fp} 1(\mathrm{~N}=37)$, Fp2 $(\mathrm{N}=36), \mathrm{F} 4(\mathrm{~N}=37), \mathrm{T} 3(\mathrm{~N}=37), \mathrm{T} 6(\mathrm{~N}=37), \mathrm{O} 1(\mathrm{~N}=36), \mathrm{Oz}(\mathrm{N}=37)$, and $\mathrm{O} 2(\mathrm{~N}=37)$, it was not possible to obtain 500 artifactfree epochs for the 38 volunteers, hence, the percentages were calculated with the number of volunteers in parentheses.

distribution presented similar detection mapping.

The overall performance of MSC in detecting response to stimulation was built in Figure 6 . The bar graphics represent the percentage of volunteers whose response to stimulation could be detected in the frequency range from 5 to $75 \mathrm{~Hz}\left(1^{\text {st }}\right.$ to $15^{\text {th }}$ multiples of the stimulation frequency $f_{e}$ ). This figure shows that only the leads $\mathrm{Cz}, \mathrm{C} 4, \mathrm{Pz}$, and $\mathrm{P} 4$ presented percents of detection higher than $70 \%$.

For lead C4, a percent of $87 \%$ was achieved for $40 \mathrm{~Hz}$, the only frequency with detection higher than $70 \%$. Percent of 82 and $71 \%$ could be seen, respectively, in the frequencies of 40 and $50 \mathrm{~Hz}$ for the parietal ipsilateral P4. However, it was in the mid-sagittal plane that the best detection rates could be found. Pz presented good performance for the frequencies $25 \mathrm{~Hz}(71 \%), 40 \mathrm{~Hz}(89 \%)$ and $50 \mathrm{~Hz}(76 \%)$. And the vertex electrode $(\mathrm{Cz})$ showed detection rates higher than $75 \%$ for the range between 25 and $50 \mathrm{~Hz}$ (except $45 \mathrm{~Hz}$ ).

\section{Discussion}

The tSEP has been widely used for monitoring purposes $(1,16-18)$ and the ORD techniques are considered a promising tool for response detection $(2,15,19)$ with a constant false-alarm rate defined a priori. However, the most suitable recording leads for tSEP is still an object of discussion.

Our results showed two main patterns of potential distribution. One presented potentials more clearly defined in the frontal, central and parietal regions, with higher amplitudes in the mid-sagittal plane and ipsilateral to the stimulated leg. The other pattern showed a wide distribution of potentials and the TSEP could be observed occupying the whole scalp surface, including the unlikely occipital electrodes, which are related with visual information processing. However, the P37-N45 was more clearly visualized in leads $\mathrm{Cz}, \mathrm{C} 4, \mathrm{Pz}$, and $\mathrm{P} 4$ for the volunteers with this 
profile. The occurrence of higher amplitude potentials ipsilateral to the stimulation is a well-known result referred to as "paradoxical lateralization" $(8,20,21)$.

The ORD results confirmed that $\mathrm{Cz}, \mathrm{Pz}, \mathrm{C} 4$, and $\mathrm{P} 4$ have higher SNR, since they presented higher MSC values and higher percentages of response detection. It is worth noting that the detection occurred for frequencies within the lower gamma band $(25-50 \mathrm{~Hz})$, which has been reported to be the maximum response band for tSEP (2).

Our results are in agreement with the IFCN report (12) suggesting that the central and parietal midsagittal regions ( $\mathrm{Cz}$ and $\mathrm{Pz}$ ) are among the best regions for response detection, even considering only unipolar derivations, instead of using $\mathrm{Cz}^{\prime}(2 \mathrm{~cm}$ behind $\mathrm{Cz}$ ) or $\mathrm{Cpz}$ (half way between $\mathrm{Cz}$ and $\mathrm{Pz}$ ) referenced to $\mathrm{Fz}$, as recommended by the IFCN Committee (12). Alternative bipolar derivations, such as [Cz'-Fpz'] (11) and [Cz-Fz] or [mid Cz/Pz-Fz] (1), are also commonly used derivations that consider the abovementioned regions as fundamental for SEP analysis.

Another bipolar derivation recommended by the IFCN report (12) is [Cc-Ci]. The recording of [C3'-C4'] (the superscript ' means $2 \mathrm{~cm}$ posterior to $\mathrm{C} 3$ and $\mathrm{C} 4$ ) for the tSEP by stimulation of the right posterior tibial nerve is suggested by Chiappa (1). Some variations such as [T3-P4] (9) were also reported as capable of registering thalamo-cortical responses due to the somatosensory stimulation (N37, P37, and N45). Using the MSC technique, the central and parietal ipsilateral regions ( $\mathrm{C} 4$ and $\mathrm{P} 4)$ to the stimulated limb resulted in detection rates from 57 to $86 \%$ in the $25-50-\mathrm{Hz}$ frequency range. Such results are better than those achieved with the contralateral leads (detection rates from 28 to $60 \%$ in the same frequency range). Considering only the contralateral leads, the central and parietal regions ( $\mathrm{C} 3$ and $\mathrm{P} 3$ ) are among the best regions for response detection. Therefore, our results agree with the IFCN recommendation of using C3'-C4', since the combination of P37 ipsilateral with N37 contralateral from the centro-parietal regions could emphasize the short latency SEP components.

In the present study, N37 was not observed or it had lower amplitude than P37 recorded at the central and parietal leads (mid-sagittal and ipsilateral to the stimuli) for all subjects. This could be caused primarily by two factors: the lower amplitude of N37 by itself and the interference by the subcortical N33 and by EMG from the temporal muscles (8).

Our results also agree with a more recent study by MacDonald et al. (22). They reported that the P37-maximum occurs more frequently at $\mathrm{CPz}$ and, in decreasing order, $\mathrm{Cz}, \mathrm{Pz}$, iCPi, CPi (where iCPi represents $\mathrm{CP} 1$ or $\mathrm{CP} 2$, and $\mathrm{CPi}$ represents $\mathrm{CP} 3$ or $\mathrm{CP} 4$, depending on which of them are the ipsilateral derivations in the International 10-10 System). Hence, the maxima occur in the central and parietal midsagittal and ipsilateral regions to the stimulated limb. For N37, the maximum was most frequently found at $\mathrm{CPC}$ (where CPc represents $\mathrm{CP} 3$ or $\mathrm{CP} 4$, depending on which was the contralateral lead) (22). Similarly, we have obtained the best detection rates (28 to $60 \%$ in the $25-50-\mathrm{Hz}$ frequency range), considering only the contralateral leads, in the central and parietal regions.

The frequency domain ORD technique permitted identification of the short-latency tSEP components with $M=500$ epochs (equivalent to $1 \mathrm{~min}$ and $40 \mathrm{~s}$ ) with stimulation at the motor threshold. MacDonald et al. (22) identified the SEP replication with $M=128$ epochs, but with stimulus at the supra-maximal intensity level.

It is well known that the highest amplitude response varies from patient to patient and can even change during surgery (23). Nevertheless, it is not a limitation of the present study because we aimed to specify the regions with the best SNR, instead of determining specific derivations. This justifies the use of unipolar derivations instead of the bipolar ones recommended by IFCN. Finally, our results showed that, using the MSC, the central and parietal regions, especially sagittal and ipsilateral to the stimuli, present the best SNR in the gamma range (tSEP characteristic) $(2,3)$.

\section{Conclusion}

Speed of detection is an important issue for surgery monitoring since it leads to more rapid changes in the surgical strategy in order to avoid neurological damage. For this application, the adequate positioning of the EEG electrodes deserves special attention. One of the requirements is acquiring the EEG signals from regions that provide higher SNR. In a medical unit, where the electromagnetic noise is more difficult to control, adequate acquisition can be much more hampered. With this reasoning, the correct selection of a suitable montage is still more critical. According to the MSC results, the leads C4, P4, $\mathrm{Cz}$, and $\mathrm{Pz}$ presented the higher SNR values. In conclusion, the parietal and central regions in the mid-sagittal line and ipsilateral to the stimulated leg can be considered as the best regions for SEP recording. Furthermore, the application of MSC may be suggested as a useful tool for monitoring purposes.

\section{Acknowledgments}

We would like to thank the Military Police Central Hospital of Rio de Janeiro for providing infrastructure support. 


\section{References}

1. Chiappa KH. Evoked potentials in clinical medicine. New York: Raven Press; 1997.

2. Infantosi AF, Melges DB, Tierra-Criollo CJ. Use of magnitude-squared coherence to identify the maximum driving response band of the somatosensory evoked potential. Braz J Med Biol Res 2006; 39: 1593-1603.

3. Tierra-Criollo CJ, Infantosi AF. Low-frequency oscillations in human tibial somatosensory evoked potentials. Arq Neuropsiquiatr 2006; 64: 402-406.

4. Simpson DM, Tierra-Criollo CJ, Leite RT, Zayen EJ, Infantosi AF. Objective response detection in an electroencephalogram during somatosensory stimulation. Ann Biomed Eng 2000; 28: 691-698.

5. Miranda de Sá AM, Infantosi AF, Simpson DM. Coherence between one random and one periodic signal for measuring the strength of responses in the electro-encephalogram during sensory stimulation. Med Biol Eng Comput 2002; 40: 99-104.

6. Miranda de Sá AM, Infantosi AF. Evaluating the entrainment of the alpha rhythm during stroboscopic flash stimulation by means of coherence analysis. Med Eng Phys 2005; 27: $167-173$

7. Valeriani M, Restuccia D, Di Lazzaro V, Barba C, Le Pera $D$, Tonali P. Dipolar generators of the early scalp somatosensory evoked potentials to tibial nerve stimulation in human subjects. Neurosci Lett 1997; 238: 49-52.

8. Valeriani M, Restuccia D, Di Lazzaro V, Barba C, Le Pera $D$, Tonali $P$. Dissociation induced by voluntary movement between two different components of the centro-parietal P40 SEP to tibial nerve stimulation. Electroencephalogr Clin Neurophysiol 1998; 108: 190-198.

9. Valeriani M, Restuccia D, Le Pera D, Barba C, Tonali P. Scalp distribution of the earliest cortical somatosensory evoked potential to tibial nerve stimulation: proposal of a new recording montage. Clin Neurophysiol 2000; 111: 14691477

10. Valeriani M, Insola A, Restuccia D, Le Pera D, Mazzone P, Altibrandi MG, et al. Source generators of the early somatosensory evoked potentials to tibial nerve stimulation: an intracerebral and scalp recording study. Clin Neurophysiol 2001; 112: 1999-2006.

11. Misulis KE. Spehlmann's evoked potential primer: Visual, auditory and somatosensory evoked potentials in clinical diagnosis. Boston: Butterworth-Heinemann; 1994.

12. Nuwer MR, Aminoff M, Desmedt J, Eisen AA, Goodin D, Matsuoka S, et al. IFCN recommended standards for short latency somatosensory evoked potentials. Report of an IFCN Committee. International Federation of Clinical Neurophysiology. Electroencephalogr Clin Neurophysiol 1994; 91: 6-11.

13. MacDonald DB, Stigsby B, Al Zayed Z. A comparison between derivation optimization and $C z^{\prime}-F P z$ for posterior tibial P37 somatosensory evoked potential intraoperative monitoring. Clin Neurophysiol 2004; 115: 1925-1930.

14. Bendat JS, Piersol AG. Random data analysis and measurement procedures. 3rd edn. New York: Wiley-Interscience; 2000.

15. Miranda de Sá AM, Infantosi AF. Evaluating the relationship of non-phase locked activities in the electroencephalogram during intermittent stimulation: a partial coherence-based approach. Med Biol Eng Comput 2007; 45: 635-642.

16. Galla JD, Ergin MA, Lansman SL, McCullough JN, Nguyen $\mathrm{KH}$, Spielvogel $\mathrm{D}$, et al. Use of somatosensory evoked potentials for thoracic and thoracoabdominal aortic resections. Ann Thorac Surg 1999; 67: 1947-1952.

17. Bose B, Sestokas AK, Schwartz DM. Neurophysiological monitoring of spinal cord function during instrumented anterior cervical fusion. Spine J 2004; 4: 202-207.

18. Jones SC, Fernau R, Woeltjen BL. Use of somatosensory evoked potentials to detect peripheral ischemia and potential injury resulting from positioning of the surgical patient: case reports and discussion. Spine J 2004; 4: 360-362.

19. Cagy M, Infantosi AF. Objective response detection technique in frequency-domain for reflecting changes in MLAEP. Med Eng Phys 2007; 29: 910-917.

20. Cruse R, Klem G, Lesser RP, Leuders H. Paradoxical lateralization of cortical potentials evoked by stimulation of posterior tibial nerve. Arch Neurol 1982; 39: 222-225.

21. Tinazzi M, Zanette G, Fiaschi A, Mauguiere F. Effect of stimulus rate on the cortical posterior tibial nerve SEPs: a topographic study. Electroencephalogr Clin Neurophysiol 1996; 100: 210-219.

22. MacDonald DB, Al Zayed Z, Stigsby B. Tibial somatosensory evoked potential intraoperative monitoring: recommendations based on signal to noise ratio analysis of popliteal fossa, optimized P37, standard P37, and P31 potentials. Clin Neurophysiol 2005; 116: 1858-1869.

23. MacDonald DB. Individually optimizing posterior tibial somatosensory evoked potential P37 scalp derivations for intraoperative monitoring. J Clin Neurophysiol 2001; 18: 364-371. 